\title{
Numerical analysis of finite source Markov retrial system with non-reliable server, collision, and impatient customers
}

\author{
Attila Kuki, Tamás Bérczes, Ádám Tóth, János Sztrik \\ University of Debrecen \\ \{kuki.attila, berczes.tamas, toth.adam, sztrik.janos\}@inf .unideb.hu \\ Submitted: February 4, 2020 \\ Accepted: July 9, 2020 \\ Published online: July 23, 2020
}

\begin{abstract}
A retrial queuing system with a single server is investigated in this paper. The server is subject to random breakdowns. The number of customers is finite and collision may take place. A collision occurs when a customer arrives to the busy server. In case of a collision both customers involved in the collision are sent back to the orbit. From the orbit the customers retry their requests after a random waiting time. The server can be down due to a failure. During the failed period the arriving customers are sent to the orbit, as well. The novelty of this analysis is the impatient behaviour of the customers. A customer waiting in the orbit may leave it after a random waiting time. The requests of these customers will not be served. All the random variables included in the model construction are assumed to be exponentially distributed and independent from each other.

The impatient property makes the model more complex, so the derivation of a direct algorithmic solution (which was provided for the non-impatient case) is difficult. For numerical calculations the MOSEL-2 tool can be used. This tool solves the Kolmogorov system equations, and from the resulting steady-state probabilities various system characteristics and performance measures can be calculated, i.e. mean response time, mean waiting time in the orbit, utilization of the server, probability of the unserved impatient requests. Principally the effect of the impatient property is investigated in
\end{abstract}


these results, which are presented graphically, as well.

Keywords: queueing, finite source, non-reliable, collision, impatient

MSC: $91 \mathrm{~B} 70$

\section{Introduction}

Retrial queueing systems (RQ-systems) are very useful tools for modeling a large variety of problems of real life situations. An RQ-system can be described by the following characteristics: when an arriving job from the outside world (from the sources) or from the queue of the system finds the server busy, joins the orbit and after a random, usually exponentially distributed time retries to reach the server again. In case of an infinite source, the orbit is assumed to be infinitely large and jobs keep retrying until they are served. The call centers, telecommunication systems, computer networks, telephone switching systems and recently smart city networks etc. can effectively be modeled by RQ-systems. Instead of the infinite source models which have been investigated by many authors, the models with finite number of sources are more appropriate to describe the behaviour of the systems under consideration. The mobile networks, sensor networks, and cognitive radio systems can be mentioned as common example of these finite source systems. The random and multiple access protocols for these types of systems have been investigated, for example in [3, 12].

In real life situations, unfortunately, the reliability of the systems cannot be assumed and assured. The elements of the systems are subject to random breakdowns. These situations also have to be investigated, so the models contain random server breakdowns and repairs. The system characteristics and performance measures are very sensitive to the non-reliable operation of the systems. Finite-source RQ-systems with server breakdowns and repairs have been investigated in several recent papers, for example in [2, 8-10, 20, 22].

The goal of this paper is to give a stochastic model for describing the phenomenon of the impatient waiting customers. The customers may retry their requests, the environment is non-reliable, and during the service process collisions might occur. A single server $M / M / 1 / / N$ retrial queueing system is useful and efficient for this task. The server is subject to random breakdowns, and the customers are subject to collisions at the service unit. This type of collisions are essential part of various implementations of telecommunication systems, computer networks. In case of busy communication channels there is large probability of conflict of signals. In these cases the signals involved into collision are lost, and retransmission is needed. The performance measures of these systems are under an optimal level. Consequently, the investigation of the systems subject to collisions has great interest nowadays. The best solution, namely building systems without collision is difficult to reach. The main effort of the investigations is to maximize the performance of the systems with collision. Previous years many authors have investigated queueing systems with conflict of customers, e.g. [1, 4, 11, 13-16, 18]. 
The novelty of this paper is the impatient behaviour of the waiting customers in the described environment, namely a single server unreliable system with a virtual waiting facility (orbit) and with possibility of collision of customers. This type of behaviour was also investigated by some authors, e.g. [5, 21]. A customer transferred to the orbit may retry its request several times. In case of unsuccessful retries after a random, exponentially distributed time the customer leaves the system (the orbit), and goes back to the source. This customer remains unserved. Our goal is to calculate the steady-state probabilities and the performance measures of these type of systems. The empirical distribution of the system probabilities and the effect of the impatient parameter are also investigated.

\section{System model}

The system under consideration is modeled by a finite source closed retrial queuing system of type $M / M / 1 / / N$. The system has one server and the number of sources is $N$. In this paper two working characteristics of the server are distinguished:

- Non-reliable server and patient customers. The server is subject to random breakdowns. The breakdown times are exponentially distributed. The breakdown parameters for busy and idle server are $\gamma_{0}$ and $\gamma_{1}$, respectively. In case of breakdown the request under service is sent to the orbit. After the breakdown the repair starts immediately. The repair time is exponentially distributed with parameter $\gamma_{2}$. While the server is under repair, the sources are able to generate requests. These customers are transferred to the orbit, because the server is not available. The requests in the orbit may retry reaching the server again after an exponentially distributed time with parameter $\sigma / N$. The customers are patient, that is they keep retrying from the orbit until they are served.

- Non-reliable server and impatient customers. The breakdown behaviour of the server is the same, as in the previous point. The customers are impatient, that is a customer keeps retrying until it is served, or the customer leaves the orbit and goes back to the source after an exponentially distributed waiting time with parameter $\tau$.

A job (customer) is generated in the source towards the server. The distribution of the inter-request times are exponential with parameter $\lambda / N$. The customer enters the system, and the source waits for a successful service. Until the end of service of the job the source cannot generate a new request. The new customer tries reaching the server. The state of the server can be busy or idle. When the server is idle, the service of the customer starts immediately. The distribution of service times is exponential with parameter $\mu$. In case of a busy server state a conflict of customers can be occur: when an arriving job finds the server busy it involves into collision with customer under service and both customers are moved into the orbit. See the model on Figure 1. 


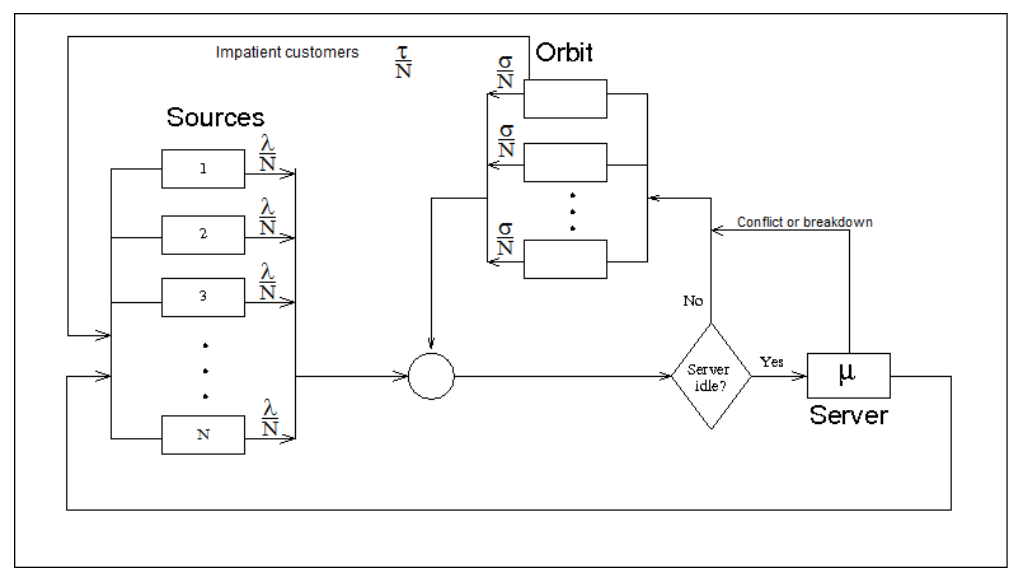

Figure 1: System model

Let $i(t)$ be the number of customers in the system. The customer can be either in the orbit or under service. Let $k(t)$ denote the status of the server:

$$
k(t)= \begin{cases}0, & \text { if the server is idle } \\ 1, & \text { if the server is busy } \\ 2, & \text { if the server is under repair. }\end{cases}
$$

Let us denote the probability that at the time t there are $i$ customers in "waiting" state and the server is in the state $k$ by $P(k(t)=k, i(t)=i)=P_{k}(i, t)$. Under the above assumption the process $X(t)=\{k(t), i(t)\}$ is a 2-dimensional Markov-chain with a state space of $\{0,1,2\} \times\{0,1, \ldots, N\}$.

The successfully served customer goes back to the source. All the random variables involved in the model construction are assumed to be totally independent from each other.

For the non-impatient case the, the Kolmogorov differential-equations for probabilities $P_{k}(i, t)$ are the following (see in $\left.[14,16]\right)$ :

$$
\begin{aligned}
\frac{\partial P_{0}(0, t)}{\partial t}= & -\left(\lambda+\gamma_{0}\right) P_{0}(0, t)+\mu P_{1}(1, t)+\gamma_{2} P_{2}(0, t), \\
\frac{\partial P_{1}(1, t)}{\partial t}= & -\left(\lambda \frac{N-1}{N}+\mu+\gamma_{1}\right) P_{1}(1, t)+\lambda P_{0}(0, t)+\frac{\sigma}{N} P_{0}(1, t), \\
\frac{\partial P_{2}(0, t)}{\partial t}= & -\left(\lambda+\gamma_{2}\right) P_{2}(0, t)+\gamma_{0} P_{0}(0, t), \\
\frac{\partial P_{0}(i, t)}{\partial t}= & -\left(\lambda \frac{N-1}{N}+\sigma \frac{i}{N}+\gamma_{0}\right) P_{0}(i, t)+\mu P_{1}(i+1, t) \\
& +\lambda \frac{N-i+1}{N} P_{1}(i-1, t)+\sigma \frac{i-1}{N} P_{1}(i, t)+\gamma_{2} P_{2}(i, t),
\end{aligned}
$$




$$
\begin{aligned}
\frac{\partial P_{1}(i, t)}{\partial t}= & -\left(\lambda \frac{N-1}{N}+\sigma \frac{i-1}{N}+\gamma_{1}+\mu\right) P_{1}(i, t) \\
& +\lambda \frac{N-i+1}{N} P_{0}(i-1, t)+\sigma \frac{i}{N} P_{0}(i, t), \\
\frac{\partial P_{2}(i, t)}{\partial t}= & -\left(\lambda \frac{N-1}{N}+\gamma_{2}\right) P_{2}(i, t)+\gamma_{0} P_{0}(i, t)+\gamma_{1} P_{1}(i, t) \\
& +\lambda \frac{N-i+1}{N} P_{2}(i-1, t) .
\end{aligned}
$$

Since $X(t)=\{k(t), i(t)\}$ is a finite state Markov-chain it can be assumed that it operates in steady-state that is: $P_{k}(i, t)=P_{k}(i)$.

Hence the steady-state Kolmogorov-equations can be written as

$$
\begin{gathered}
-\left(\lambda+\gamma_{0}\right) P_{0}(0)+\mu P_{1}(1)+\gamma_{2} P_{2}(0)=0, \\
-\left(\lambda \frac{N-1}{N}+\mu+\gamma_{1}\right) P_{1}(1)+\lambda P_{0}(0)+\frac{\sigma}{N} P_{0}(1)=0 \\
-\left(\lambda+\gamma_{2}\right) P_{2}(0)+\gamma_{0} P_{0}(0)=0, \\
-\left(\lambda \frac{N-1}{N}+\sigma \frac{i}{N}+\gamma_{0}\right) P_{0}(i)+\mu P_{1}(i+1)+\lambda \frac{N-i+1}{N} P_{1}(i-1) \\
+\sigma \frac{i-1}{N} P_{1}(i)+\gamma_{2} P_{2}(i)=0, \\
-\left(\lambda \frac{N-1}{N}+\sigma \frac{i-1}{N}+\gamma_{1}+\mu\right) P_{1}(i)+\lambda \frac{N-i+1}{N} P_{0}(i-1)+\sigma \frac{i}{N} P_{0}(i)=0, \\
-\left(\lambda \frac{N-1}{N}+\gamma_{2}\right) P_{2}(i)+\gamma_{0} P_{0}(i)+\gamma_{1} P_{1}(i)+\lambda \frac{N-i+1}{N} P_{2}(i-1)=0 .
\end{gathered}
$$

Note, if all of the $\gamma_{2}$ parameters and $P_{2}$ probabilities are set to zero, we get the formulas for the system with conflict and reliable server.

By the help of the same method described above, the steady-state Kolmogorovequations can be obtained for the system with conflict, non-reliable server and impatient customers:

$$
\begin{gathered}
-\left(\lambda+\gamma_{0}\right) P_{0}(0)+\mu P_{1}(1)+\gamma_{2} P_{2}(0)+\frac{\tau}{n} P_{0}(1)=0 \\
-\left(\lambda \frac{N-1}{N}+\mu+\gamma_{1}\right) P_{1}(1)+\lambda P_{0}(0)+\frac{\sigma}{N} P_{0}(1)+\frac{\tau}{n} P_{1}(2)=0 \\
-\left(\lambda+\gamma_{2}\right) P_{2}(0)+\gamma_{0} P_{0}(0)=0 \\
-\left(\lambda \frac{N-i}{N}+\sigma \frac{i}{N}+\tau \frac{i}{N}+\gamma_{0}\right) P_{0}(i)+\mu P_{1}(i+1)+\lambda \frac{N-i+1}{N} P_{1}(i-1) \\
+\sigma \frac{i-1}{N} P_{1}(i)+\tau \frac{i+1}{N} P_{0}(i+1)+\gamma_{2} P_{2}(i)=0 \\
-\left(\lambda \frac{N-i}{N}+\sigma \frac{i-1}{N}+\tau \frac{i-1}{N}+\gamma_{1}+\mu\right) P_{1}(i)+\lambda \frac{N-i+1}{N} P_{0}(i-1)
\end{gathered}
$$




$$
\begin{gathered}
+\sigma \frac{i}{N} P_{0}(i)+\tau \frac{i}{N} P_{1}(i+1)=0 \\
-\left(\lambda \frac{N-i}{N}+\gamma_{2}\right) P_{2}(i)+\gamma_{0} P_{0}(i)+\gamma_{1} P_{1}+\lambda \frac{N-i+1}{N} P_{2}(i-1) \\
+\tau \frac{i+1}{N} P_{2}(i+1)=0
\end{gathered}
$$

\section{Performance Measures}

The performance measures express the effect of the input parameters of the system. Let us define the most important characteristics which can be determined directly from the steady state probabilities.

- Mean number of customers in the system $\bar{Q}$ and in the orbit $\bar{O}$

$$
\bar{Q}=\sum_{i=0}^{N} i P(i), \quad \bar{O}=\bar{Q}-P_{1},
$$

- Mean arrival rate $\bar{\lambda}$

$$
\bar{\lambda}=\sum_{k=0}^{1} \sum_{i=0}^{N}(N-i) \frac{\lambda}{N} P_{k}(i),
$$

- Mean response time $\bar{T}$ and mean waiting time $\bar{W}$ in the orbit can be obtained by the Little-formula

$$
\bar{T}=\frac{\bar{Q}}{\bar{\lambda}}, \quad \bar{W}=\frac{\bar{O}}{\bar{\lambda}}, \quad \bar{O}=\bar{Q}-P_{1},
$$

- Mean total service time $E\left(T_{S}\right)$ and mean total sojourn time in the source $E(\kappa)$

$$
E\left(T_{S}\right)=\bar{T}-\bar{W}, \quad E(\kappa)=\frac{(N-\bar{Q}) \bar{T}}{\bar{Q}},
$$

- Mean number of trials from the source $E\left(N_{T S}\right)$ and from the orbit $E\left(N_{T O}\right)$

$$
E\left(N_{T S}\right)=\frac{\lambda}{N} E(\tau), \quad E\left(N_{T O}\right)=\frac{\sigma}{N} \bar{W}
$$

\section{Numerical solution}

Before this model several other systems were investigated. Simple retrial queueing models, retrial models with conflict of customers, non-reliable retrial models with conflict of customers, retrial models with two-way communications. Obtaining the 
system characteristics three different solutions were performed: recursive numerical calculations, solving the system equations (e.g. by MOSEL-2 tool), and run simulations. The results of the three different approaches were identical. Using these results, we were able to investigate models, where all of the mentioned solutions were not applicable. For example, systems with non-exponentially distributed service times can not be solved by MOSEL-2, but simulation and, in some cases, numerical solution proved useful.

The situation for this model is very similar. For the non-impatient case the equations can be solved recursively (described in $[14,16,19])$. The resulting steadystate probabilities $P_{k}(i)$ can be used for calculating the system performance measures. For double-checking the result, MOSEL-2 tool can also be applied here. For the impatient case we did our best, but such recursive solution cannot be obtained, because new variables enter into equations due to the impatient property. For this impatient case a software tool, MOSEL-2 is used to solve the system equations. The correctness of MOSEL-calculations was empirically proved in cases, when this tool and the numerical calculations were used simultaneously.

On Figure 2 the steady-state probabilities are displayed for the different models (non-conflict, conflict, unreliable, unreliable block, unreliable impatient). When the calculations are performed by MOSEL-2 tool (MOdeling Specification and Evaluation Language), see in [6], we run into a strict limitations, namely the state space grows extremely fast, consequently the number of sources cannot exceed 200 . In Excel we can go far more above 200 (when the recursive calculations can be performed.

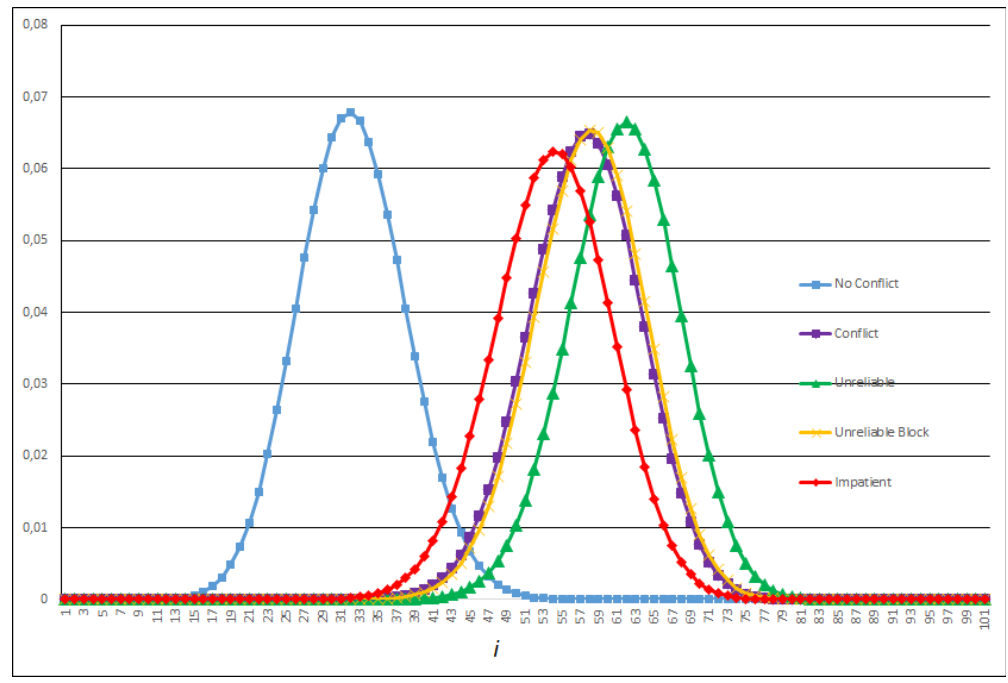

Figure 2: Different models

The first important question was the distribution of the system probabilities $P_{k}(i)$. Previous investigations the distribution was found very close to normal dis- 
tribution. The normality is important, because in general only the average number of customers in the orbit or average waiting time of customers in the orbit can be calculated by the methods mentioned in this paper. But, if the normality of steady state probabilities can be assumed, the limiting probability distribution of the sojourn time/waiting time of the customer in the orbit can be obtained by asymptotic methods. See in $[7,17]$. That's why is it important to find domains of parameters, where the steady-state system (or orbit) probabilities have normal or asymptotic normal distribution. Here the normality of the distribution was checked for different numbers of sources: $N=50,100$ and 200. Then the Kolmogorov-distance was computed. For the Kolmogorov-distance the theoretical normal distribution is calculated by using Excel built-in function. The parameters of the distribution is calculated from the steady-state probabilities. For example, in case of $N=100$, a normal distribution is generated with mean of 52.9 and standard deviation of 6.37 . The Kolmogorov-distance is defined as:

$$
\Delta_{N}=\max _{0 \leq k \leq N}\left|\sum_{i=0}^{k} P_{\text {Theoretical }}(i)-\sum_{i=0}^{k} P_{\text {Mosel }}(i)\right| .
$$

The following result were found: $\Delta_{50}=0.03, \Delta_{100}=0.02, \Delta_{200}=0.003$. Thus the normality of the system probabilities can be accepted.

On Figure 3 the cumulative distribution function (CDF) of the normal (Gaussian) distribution and the empirical CDF are compared. As from the Kolmogorovdistance can be expected, the two distributions are almost identical.

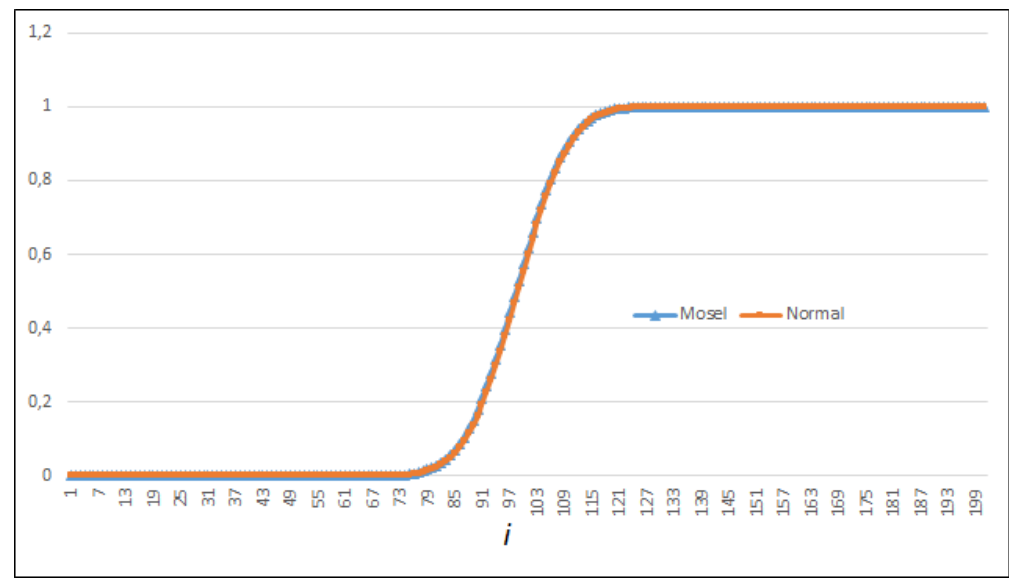

Figure 3: Normal CDF vs. empirical CDF

As described above, from the steady-state probabilities the performance measures (system characteristics) can be calculated.

On Figure 4 the mean response time, calculated by the help of formulas presented in Chapter 3 is displayed as a function of the overall generation rate. The 


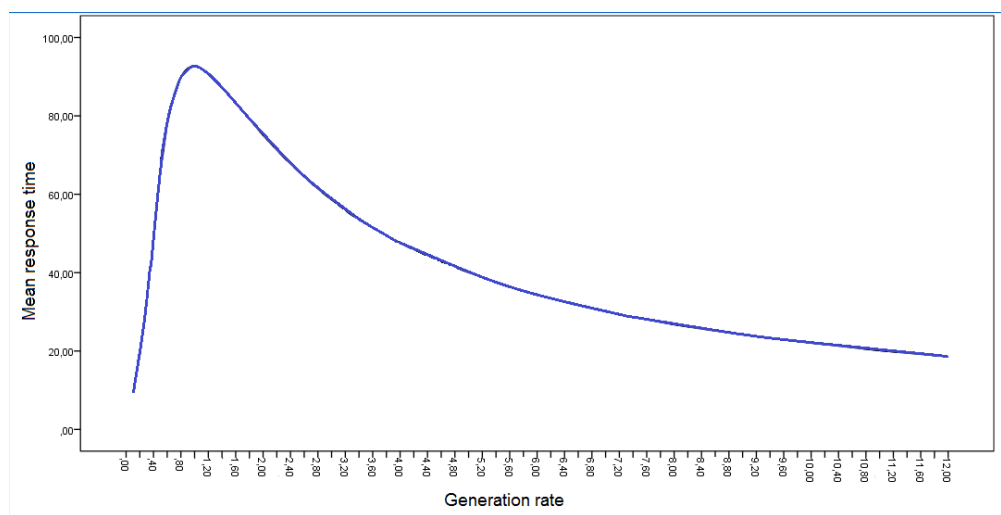

Figure 4: Mean response time vs. $\lambda$

expected maximum characteristic can be observed on this figure, as well. Under some parameter settings the finite-source retrial queueing systems have this maximum feature for several performance measures, e.g. response time. The reason is the special coincidence of the high generation rate and the low number of active tokens in the source (the number of jobs in the system is usually high at this situation).

\section{Conclusion}

The goal of this paper was to handle the impatient behaviour of customers in the environment of unreliable systems with collision. For non-impatient systems computing the steady-state system characteristics a recursive solution can be given. The impatient property makes the system equations more complex. New variables appear in the equations, so the recursive numeric solution cannot be performed. Because of this reason a software tool was used to solve the system equations. For this complex case there is no limit distribution of sojourn and waiting times of customers in the orbit. So, it is important to find domains of parameter, where the distribution of steady-state probabilities can be accepted as normal, to give the possibility of further theoretical investigations towards the limit distributions.

Acknowledgements. The research work was supported by the construction EFOP - 3.6.3 - VEKOP - 16-2017-00002. The project was supported by the European Union, co-financed by the European Social Fund. 


\section{References}

[1] A.-A. ALI, S. WeI: Modeling of coupled collision and congestion in finite source wireless access systems, in: Wireless Communications and Networking Conference (WCNC), 2015 IEEE, IEEE, 2015, pp. 1113-1118.

[2] B. Almási, J. Roszik, J. Sztrik: Homogeneous finite-source retrial queues with server subject to breakdowns and repairs. English, Math. Comput. Modelling 42.5-6 (2005), pp. 673682, ISSN: 0895-7177,

DOI: $10.1016 / \mathrm{j} . \mathrm{mcm} \cdot 2004.02 .046$.

[3] J. Artalejo, A. G. Corral: Retrial Queueing Systems: A Computational Approach, Springer, 2008.

[4] S. Balsamo, G.-L. Dei Rossi, A. Marin: Modelling retrial-upon-conflict systems with product-form stochastic Petri nets, in: International Conference on Analytical and Stochastic Modeling Techniques and Applications, Springer, 2013, pp. 52-66.

[5] D. BARRER: Queuing with impatient customers and ordered service, Operation Research 5 (1957), pp. 650-656.

[6] T. Bérczes, J. Sztrik, Á. Tóth, A. Nazarov: Performance Modeling of Finite-Source Retrial Queueing Systems with Collisions and Non-reliable Server Using MOSEL, in: International Conference on Distributed Computer and Communication Networks, Springer, 2017, pp. 248-258.

[7] E. Y. Danilyuk, S. P. Moiseeva, J. Sztrik: Asymptotic Analysis of Retrial Queueing System $M / M / 1$ with Impatient Customers, Collisions and Unreliable Server, Journal of Siberian Federal University. Mathematics \& Physics 13.2 (2020), pp. 218-230, DOI: $10.17516 / 1997-1397-2020-13-2-218-230$.

[8] V. I. Dragieva: Number of retrials in a finite source retrial queue with unreliable server. English, Asia-Pac. J. Oper. Res. 31.2 (2014), p. 23, Issn: 0217-5959; 1793-7019/e, DOI: $10.1142 / \mathrm{S} 0217595914400053$.

[9] N. Gharbi, C. Dutheillet: An algorithmic approach for analysis of finite-source retrial systems with unreliable servers, English, Computers \& Mathematics with Applications 62.6 (2011), pp. 2535-2546, ISSN: 0898-1221,

DOI: $10.1016 / \mathrm{j}$. camwa.2011.03.109.

[10] A. Gómez-Corral, T. Phung-Duc: Retrial queues and related models, Annals of Operations Research 247.1 (2016), pp. 1-2, IssN: 1572-9338, DOI: $10.1007 / \mathrm{s} 10479-016-2305-2$.

[11] J. S. Kıм: Retrial queueing system with collision and impatience, Communications of the Korean Mathematical Society 25.4 (2010), pp. 647-653.

[12] J. Kıм, B. Kıм: A survey of retrial queueing systems, Annals of Operations Research 247.1 (2016), pp. 3-36, Issn: 1572-9338, DOI: $10.1007 / \mathrm{s} 10479-015-2038-7$.

[13] A. Kuki, T.Bérczes, J. Sztrik, A. Kvach: Numerical Analysis of Retrial Queueing Systems with Conflict of Customers, Journal of Mathematical Sciences (2017), submitted.

[14] A. Kvach, A. Nazarov: Sojourn Time Analysis of Finite Source Markov Retrial Queuing System with Collision, in: Information Technologies and Mathematical Modelling - Queueing Theory and Applications: 14th International Scientific Conference, ITMM 2015, named after A. F. Terpugov, Anzhero-Sudzhensk, Russia, November 18-22, 2015, Proceedings, Cham: Springer International Publishing, 2015, chap. 8, pp. 64-72.

[15] T. V. Lyubina, A. A. Nazarov: Research of the non-Markov dynamic retrial queue system with collision (In Russian), Herald of Kemerovo State University 1.49 (2012), pp. 38-44. 
[16] A. Nazarov, A. Kvach, V. Yampolsky: Asymptotic Analysis of Closed Markov Retrial Queuing System with Collision, in: Information Technologies and Mathematical Modelling: 13th International Scientific Conference, ITMM 2014, named after A.F. Terpugov, AnzheroSudzhensk, Russia, November 20-22, 2014. Proceedings, Cham: Springer International Publishing, 2014, chap. 1, pp. 334-341, ISBN: 978-3-319-13671-4.

[17] A. Nazarov, J. Sztrik, A. Kvach, Á. Tóth: Asymptotic sojourn time analysis of finitesource $M / M / 1$ retrial queueing system with collisions and server subject to breakdowns and repairs, Annals of Operations Research 288.1 (2019), pp. 417-434, DOI: $10.1007 / \mathrm{s} 10479-019-03463-0$.

[18] Y. Peng, Z. Liu, J. Wu: An M/G/1 retrial G-queue with preemptive resume priority and collisions subject to the server breakdowns and delayed repairs. English, J. Appl. Math. Comput. 44.1-2 (2014), pp. 187-213, ISSN: 1598-5865; 1865-2085/e, DOI: $10.1007 / \mathrm{s} 12190-013-0688-7$.

[19] Á. Tóth, T. Bérczes, J. Sztrik, A. Kuki: Comparison of two operation modes of finitesource retrial queueing systems with collisions and non-reliable server by using simulation, Journal of Mathematical Sciences 237 (2019), pp. 846-857.

[20] J. WANG, L. ZHAO, F. ZHANG: Analysis of the finite source retrial queues with server breakdowns and repairs, English, Journal of Industrial and Management Optimization 7.3 (2011), pp. 655-676, ISSN: 1547-5816; 1553-166X/e, DOI: $10.3934 / \mathrm{jimo} .2011 .7 .655$.

[21] P. Wüchner, J. Sztrik, H. De Meer: Finite-source $M / M / S$ retrial queue with search for balking and impatient customers from the orbit, Computer Networks 53.8 (2009), pp. 12641273.

[22] F. Zhang, J. WANG: Performance analysis of the retrial queues with finite number of sources and service interruptions, English, Journal of the Korean Statistical Society 42.1 (2013), pp. 117-131, ISSN: 1226-3192,

DOI: $10.1016 / j \cdot j k s s .2012 .06 .002$. 